\title{
Inflammasome might contribute to impaired fertility of men with spinal cord injury
}

Infertility is a serious issue for men who have suffered spinal cord injury (SCI). As well as erectile and ejaculatory dysfunction resulting from neurological trauma, injured men also demonstrate low sperm motility and viability, the reasons for which are unclear. Newly published research from the Miami Project to Cure Paralysis suggests the inflammasome might be involved.

Identifying the mechanisms responsible for poor-quality sperm in men with SCI was not easy. "Our early investigations examined lifestyle factors, such as infrequency of ejaculation and elevated scrotal temperature from sitting in a wheelchair, as well as time since injury and frequent UTIs, but none of these factors could explain the problem," remarks Emad Ibrahim, corresponding author of the study. A breakthrough came when the researchers discovered it was the seminal plasma that was abnormal in these patients, containing high levels of inflammatory cytokines that exert a toxic effect on sperm.

The inflammasome is a multiprotein complex that has been termed the 'master switch' of the innate immune response. Armed with this knowledge, Ibrahim and colleagues explored the expression of its component proteins caspase- 1 and apoptosis-associated speck-like protein containing a caspase activation and recruitment domain (ASC) in the seminal plasma of men with SCI.

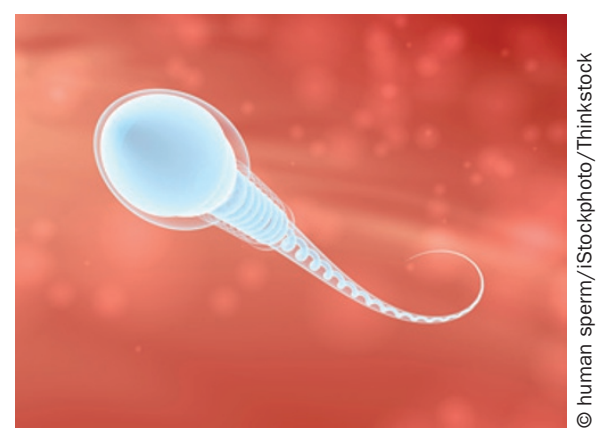

Expression levels of both ASC and caspase-1 were significantly higher (9.1-fold and sixfold, respectively) in men with SCI $(n=28)$ than in age-matched healthy volunteers $(n=28)$, and linear regression analysis revealed a negative correlation between ASC levels and sperm motility. Further analysis of semen samples almost free of leukocytes excluded the contribution of leukocytospermia to these findings. Finally, confocal microscopy confirmed the expression of inflammasome proteins in sperm.

Ibrahim and his team are currently investigating whether anti-inflammasome therapies, such as antibody neutralization, might improve fertility in these men.

\section{Sarah Payton}

Original article Zhang, X. et al. Involvement of the inflammasome in abnormal semen quality of men with spinal cord injury. Fertil. Steril. doi:10.1016/j. fertnstert.2012.09.004 